\title{
Interação genótipo $\times$ ambiente para produção de leite de bovinos da raça Holandesa entre bacias leiteiras no estado do Paraná
}

\author{
Meiby Carneiro de Paula ${ }^{1}$, Elias Nunes Martins ${ }^{1}$, Luiz Otávio Campos da Silva ${ }^{2}$, \\ Carlos Antonio Lopes de Oliveira ${ }^{3}$, Altair Antônio Valotto ${ }^{4}$, Newton Pohl Ribas ${ }^{5}$
}

\author{
${ }^{1}$ Departamento de Zootecnia da Universidade Estadual de Maringá - PR. \\ 2 Embrapa Gado de Corte - Campo Grande. \\ 3 Universidade Estadual do Mato Grosso do Sul. \\ 4 Serviço de Registro Genealógico da Associação Paranaense de Criadores de Bovinos da Raça Holandesa. \\ ${ }^{5}$ Universidade Federal do Paraná - UFPR.
}

RESUMO - Foram analisados 117.082 registros de lactações encerradas para a produção de leite corrigida para os 305 dias de lactação (PL305) de 49.676 vacas da raça Holandesa, provenientes de 308 rebanhos distribuídos em sete bacias leiteiras no estado do Paraná, com o objetivo de verificar a existência de interação genótipo $\times$ ambiente para a PL305 desses animais utilizando-se a inferência Bayesiana. Todos os animais foram controlados oficialmente entre janeiro de 1992 a dezembro de 2003 pelo Serviço de Controle Leiteiro Mensal da Associação Paranaense de Criadores de Bovinos da Raça Holandesa. Os componentes de co-variância e os parâmetros genéticos foram estimados por meio de análises uni e multicaracteres, de modo que, na análise multicaracter, a PL305 em cada uma das bacias foi tratada como uma característica diferente. A produção de leite corrigida para os 305 de lactação, em kg, nas bacias leiteiras de Castro, Carambeí, Witmarsum, Arapoti, Sul, Norte e Oeste foram de 8.414 $\pm 1.825,8.481 \pm 2.010,7.636 \pm 1.594,7.850 \pm 1.795$, $8.617 \pm 2.050,7.401 \pm 1.809$ e $7.336 \pm 2.456$, respectivamente. A estimativa de herdabilidade mais alta $(0,39)$ foi obtida para a bacia leiteira do Oeste e a mais baixa $(0,23)$ para a de Carambeí. As correlações genéticas obtidas entre as bacias leiteiras foram baixas $(0,09$ a 0,57$)$. As correlações de Pearson e de Spearman mais baixas foram obtidas para a bacia leiteira do Oeste do Paraná e variaram de 0,37 a 0,41 e de 0,37 a 0,49 , respectivamente. Esses resultados comprovam a existência de interação genótipo $\times$ ambiente nas bacias leiteiras do estado do Paraná.

Palavras-chave: avaliação genética, bovinos de leite, componentes de variância, correlação genética, herdabilidade, inferência Bayesiana

\section{Genotype x environment interaction for milk yield of Holstein cows among dairy production units in the state of Paraná}

\footnotetext{
ABSTRACT - It was analyzed 117,082 complete lactation records adjusted for 305 days in milk yield (MY305) of 49,676 Holsteins cows from 308 herds distributed among seven dairy production units in the state of Paraná, Brazil. The objective was to verify the genotype $\times$ environment interaction to MY305 of Holsteins cows between dairy production units in the state of Paraná using the Bayesian inference. All animals were officially controlled by the Serviço de Controle Leiteiro Mensal of Associação Paranaense de Criadores de Bovinos da Raça Holandesa, between January/1992 and December/2003. The (co)variance components and genetic parameters were estimated using one and multiple-trait analysis, where MY305 of each dairy production unit was considered as a different variable. Means and standard deviations for MY305 (kg) for dairy production units of Castro, Carambeí, Witmarsum, Arapoti, Sul, Norte and Oeste, were 8,414 $\pm 1,825 ; 8,481$ $\pm 2,010 ; 7,636 \pm 1,594 ; 7,850 \pm 1,795 ; 8,617 \pm 2,050 ; 7,401 \pm 1,809$ and 7,336 $\pm 2,456$, respectively. The highest heritability estimated was obtained for the Oeste unit (0.39) while Carambeí unit presented the lowest value (0.23). Genetic correlations were low (0.09 to 0.57 ) between dairy production units. Pearson and Spearman correlations were lower for the Oeste unit, when compared with other dairy production units, which ranged from 0.37 to 0.41 , and from 0.37 to 0.49 , respectively. These results show the presence of genotype $\times$ environment interaction between dairy production units in the state of Paraná.
}

Key Words: Bayesian inference, dairy cattle, genetic correlation, genetic evaluation, heritability, variance components

Este artigo foi recebido em 18/1/2006 e aprovado em 13/8/2008.

Correspondências devem serenviadas para:meibydepaula@hotmail.com 


\section{Introdução}

Tanto a genética como o ambiente determinam a expressão das características de importância econômica de bovinos de leite. Entretanto, alguns genótipos podem não se expressar da mesma maneira em diferentes ambientes em virtude da interação genótipo $\times$ ambiente.

A interação genótipo $\times$ ambiente pode provocar alterações nas variações genéticas, fenotípicas e ambientais e, conseqüentemente, modificar as estimativas dos parâmetros genéticos e fenotípicos, portanto, dependendo do ambiente, há possibilidade de mudanças nos critérios de seleção, de modo que a identificação dessas interações contribua para o aumento da eficiência da seleção em bovinos (Alencar et al., 2005).

A existência de interação genótipo $\times$ ambiente para a produção de leite em bovinos da raça Holandesa tem sido observada em alguns estudos em diversos países (Stanton et al., 1991; Costa et al., 2000; Zwald et al., 2003; CerónMuñoz et al., 2004; Rekaya et al., 2003) e, no Brasil, entre diferentes estados (Falcão et al., 2006). Entretanto, avaliações em regiões menores, como dentro de um mesmo estado, ainda não foram realizadas no Brasil.

O estado do Paraná se destaca por apresentar rebanhos com alto potencial genético para produção de leite, é o terceiro maior produtor de leite do País (2,5 bilhões de litros), com produtividade média de 1.660 litros/vacas/ano, superior à média nacional (SEAB-DERAL, 2007). As diferenças nas condições de clima, manejo, produção e constituição dos rebanhos neste estado podem levar à interação genótipo $\times$ ambiente.

Este trabalho foi realizado para verificar a existência de interação genótipo $\times$ ambiente para a produção de leite de bovinos da raça Holandesa entre bacias leiteiras do estado do Paraná utilizando-se a inferência Bayesiana.

\section{Material e Métodos}

Foram disponibilizados 195.886 registros de lactações encerradas de vacas da raça Holandesa pelo Programa de Análise de Rebanhos Leiteiros do Paraná (PARLPR), desenvolvido em convênio com a Associação Paranaense de Criadores de Bovinos da Raça Holandesa (APCBRH), localizada em Curitiba, Paraná. Todos os animais foram controlados oficialmente pelo Serviço de Controle Leiteiro Mensal da APCBRH, entre janeiro de 1992 a dezembro de 2003.

Para garantir a consistência das informações, o conjunto de dados original foi editado com o uso do programa computacional Statistical Analysis System (SAS, 2000), eliminando-se observações inconsistentes. Também foram eliminadas informações de vacas cujos pais tinham menos de oito filhas ou procediam de rebanhos com menos de dez lactações.

Foram definidas duas estações de parto (uma do mês de abril a setembro e outra do mês de outubro a março) e criados grupos de contemporâneos (GC) formados por lactações de mesmo rebanho, de animais que pariram no mesmo ano em mesma estação de parto. Foram mantidas apenas informações pertencentes a grupos de contemporâneos com dez ou mais observações.

Após a aplicação das restrições, o conjunto final de dados permaneceu com 117.082 registros da $1^{\mathrm{a}}$ a $10^{\mathrm{a}}$ lactação para produção de leite corrigida para os 305 dias de lactação (PL305), provenientes de 308 rebanhos distribuídos em sete bacias leiteiras do estado do Paraná: Castro, Carambeí, Witmarsum, Arapoti, Sul, Norte e Oeste (Tabela 1).

A divisão do estado em sete bacias leiteiras foi feita segundo critérios da APCBRH, que agrupa os rebanhos de acordo com o total de leite recebido pelas cooperativas em que cada rebanho é associado. Cooperativas que recebem grande volume de leite, mesmo estando na mesma região geográfica, foram consideradas bacias leiteiras diferentes, independentemente de sua localização. Assim, os municípios de Carambeí, Castro, Witmarsum e Arapoti, pertencentes à região de Ponta-Grossa, região sul do Paraná, foram divididos em quatro bacias leiteiras diferentes, com os mesmos nomes. As outras cooperativas da região sul foram agrupadas em uma só, chamada Bacia Leiteira do Sul. As demais bacias leiteiras foram estabelecidas conforme a localização do município - sede dos laticínios de cada região geográfica do estado.

A região sul apresenta clima mesotérmico, úmido e superúmido, sem estação seca, com inverno rigoroso, geadas severas e freqüentes, verões chuvosos e amenos. A região norte apresenta clima mesotérmico, sem estação seca, verões quentes e baixa incidência de geadas. A região

Tabela 1 - Número de lactações, vacas, rebanhos, grupos de contemporâneos

\begin{tabular}{lrrccc}
\hline $\begin{array}{l}\text { Bacia } \\
\text { leiteira }\end{array}$ & Lactação & Vaca & Rebanho & $\begin{array}{c}\text { Grupo de } \\
\text { contemporâneo }\end{array}$ & Touro \\
\hline Castro & 42.128 & 17.032 & 58 & 969 & 849 \\
Carambeí & 27.289 & 12.108 & 97 & 1.095 & 823 \\
Witmarsum & 11.385 & 4.533 & 35 & 414 & 473 \\
Arapoti & 22.953 & 9.286 & 37 & 616 & 681 \\
Sul & 5.227 & 2.424 & 22 & 183 & 371 \\
Norte & 6.091 & 3.168 & 41 & 256 & 387 \\
Oeste & 2.009 & 1.125 & 18 & 122 & 329 \\
Total & 117.082 & 49.676 & 308 & 3.655 & - \\
\hline
\end{tabular}

(C) 2009 Sociedade Brasileira de Zootecnia 
oeste apresenta clima mesotérmico, sem estação seca, com inverno rigoroso, média incidência de chuvas e ocorrência de geadas, verões chuvosos e temperatura elevada (SEABDERAL, 2000).

A estimação dos componentes de co-variância para PL305 e dos parâmetros genéticos foi realizada utilizando-se abordagem Bayesiana, por meio do programa MTGSAM (Multiple Trait Gibbs Sampling in Animal Models) desenvolvido por Van Tassel \& Van Vleck (1995). Os dados foram analisados por análise unicaracter, considerando a PL305 nas diferentes bacias leiteiras uma característica única, e por análise multicaracter, considerando a PL305 em cada uma das sete bacias leiteiras uma característica diferente. Tanto para a análise unicaracter como para a multicaracter, foi utilizado um modelo animal que incluiu os efeitos fixos de grupo de contemporâneos, número de ordenhas e efeito linear e quadrático da co-variável idade da vaca ao parto, em meses. Os efeitos aleatórios considerados foram os genéticos aditivos, permanente de ambiente e erro residual. Utilizou-se o modelo animal descrito abaixo:

$$
y=X \beta+Z a+W p+e
$$

em que: $y=$ vetor de observações da PL305; $\beta=$ vetor dos efeitos fixos; $a, p, e=$ vetores dos efeitos genéticos aditivos, permanente de ambiente e dos erros aleatórios, respectivamente. $X, Z$ e $W=$ matrizes de incidência dos efeitos fixos, genéticos aditivos e permanente de ambiente, respectivamente. Admitiu-se que $y, a, p$ e $e$ possuem distribuição conjunta normal multivariada, de modo que:

$$
\left[\begin{array}{c}
y \\
a \\
p \\
e
\end{array}\right] \sim N M V\left\{\left[\begin{array}{c}
X \beta \\
\phi \\
\phi \\
\phi
\end{array}\right],\left[\begin{array}{cccc}
Z G Z^{\prime}+W P W^{\prime}+R & Z G & W P & R \\
G Z^{\prime} & G & \phi & \phi \\
P W^{\prime} & \phi & P & \phi \\
R & \phi & \phi & R
\end{array}\right]\right\}
$$

Na análise unicaracter, $\mathrm{G}=\mathrm{A} \sigma_{a}^{2}, \mathrm{P}=I_{m} \sigma_{p}^{2} \mathrm{e} \mathrm{R}=I_{q} \sigma_{e}^{2}$, em que $A=$ matriz de parentesco entre os animais; $\sigma_{a}^{2}, \sigma_{p}^{2}$, $\sigma_{e}^{2}=$ variância genética aditiva, permanente de ambiente e residual, respectivamente; $I_{m}=$ matrizidentidade, de ordem $m$ ( $m$ igual ao número de vacas); $I_{q}=$ matriz identidade, de ordem $q$ ( $q$ igual ao número de lactações); $Z$ e $W=$ matrizes de incidência dos valores genéticos e dos efeitos permanentes de ambiente, respectivamente.

$\mathrm{y}=$ Em termos matriciais, o modelo animal utilizado n análise multicaracter foi:

$$
\begin{aligned}
\mathrm{y} & =\left[\begin{array}{c}
y_{1} \\
y_{2} \\
\vdots \\
y_{7}
\end{array}\right]=\left[\begin{array}{cccc}
X_{1} & 0 & \cdots & 0 \\
0 & X_{2} & \cdots & 0 \\
\vdots & \vdots & \ddots & \vdots \\
0 & 0 & \cdots & X_{7}
\end{array}\right] \cdot\left[\begin{array}{c}
\beta_{1} \\
\beta_{2} \\
\vdots \\
\beta_{7}
\end{array}\right]+\left[\begin{array}{cccc}
Z_{1} & 0 & \cdots & 0 \\
0 & Z_{2} & \cdots & 0 \\
\vdots & \vdots & \ddots & \vdots \\
0 & 0 & \cdots & Z_{7}
\end{array}\right] \cdot\left[\begin{array}{c}
a_{1} \\
a_{2} \\
\vdots \\
a_{7}
\end{array}\right]+ \\
& {\left[\begin{array}{cccc}
W_{1} & 0 & \cdots & 0 \\
0 & W_{2} & \cdots & 0 \\
\vdots & \vdots & \ddots & \vdots \\
0 & 0 & \cdots & W_{7}
\end{array}\right] \cdot\left[\begin{array}{c}
p_{1} \\
p_{2} \\
\vdots \\
p_{7}
\end{array}\right]+\left[\begin{array}{c}
e_{1} \\
e_{2} \\
\vdots \\
e_{7}
\end{array}\right] }
\end{aligned}
$$

em que $y_{1}, y_{2}, y_{3}, y_{4}, y_{5}, y_{6}$ e $y_{7}$ são os vetores de observações da PL305; $X_{1}, X_{2}, X_{3}, X_{4}, X_{5}, X_{6}$ e $X_{7}$, as matrizes de incidência dos efeitos fixos, contidas nos vetores $\beta_{1}, \beta_{2}, \beta_{3}, \beta_{4}, \beta_{5}, \beta_{6}$ e $\beta_{7}$, respectivamente; $Z_{1}, Z_{2}, Z_{3}, Z_{4}, Z_{5}, Z_{6}$ e $Z_{7}$, as matrizes de incidência dos efeitos genéticos aditivos, contidas nos vetores $a_{1}, a_{2}, a_{3}$, $a_{5}, a_{6}$ e $a_{7}$, respectivamente; $W_{1}, W_{2}, W_{3}, W_{4}, W_{5}, W_{6}$ e $W_{7}$, as matrizes de incidência dos efeitos permanentes de ambiente, contidas nos vetores $p_{1}, p_{2}, p_{3}, p_{4}, p_{5}, p_{6}$ e $p_{7}$, respectivamente; $e_{1}, e_{2}, e_{3}, e_{4}, e_{5}, e_{6} \mathrm{e} e_{7}$, os vetores dos erros aleatórios, associados aos vetores $y_{1}, y_{2}, y_{3}, y_{4}, y_{5}$, $y_{6}$ e $y_{7} ; 1,2,3,4,5,6$ e 7, as bacias leiteiras de Castro, Carambeí, Witmarsum, Arapoti, Sul, Norte e Oeste, respectivamente.

$\mathrm{Na}$ análise multicaracter, as matrizes $G, P$ e $R$ foram definidas como $G=G_{0} \otimes A, P=\bigoplus_{i=1}^{7} I_{m_{i}} \sigma_{p_{i}}^{2}$ e $R=\bigoplus_{i=1}^{7} I_{q_{i}} \sigma_{e_{i}}^{2}$, respectivamente, em que $G_{0}=$ matriz de co-variância genética aditiva das sete bacias leiteiras, determinada por:

$$
G_{0}=\left[\begin{array}{cccc}
\sigma_{a_{1}}^{2} & \sigma_{a_{1} a_{2}} & \cdots & \sigma_{a_{1} a_{7}} \\
\sigma_{a_{2} a_{1}} & \sigma_{a_{2}}^{2} & \cdots & \sigma_{a_{2} a_{7}} \\
\vdots & \vdots & \ddots & \vdots \\
\sigma_{a_{7} a_{1}} & \sigma_{a_{7} a_{2}} & \cdots & \sigma_{a_{7}}^{2}
\end{array}\right]
$$

em que $\sigma_{a_{i}}^{2}, \sigma_{p_{i}}^{2}$ e $\sigma_{e_{i}}^{2}=$ variância genética aditiva, permanente de ambiente e residual, respectivamente, das $i$ bacias leiteiras; e $\sigma_{a_{i} a_{j}}=$ co-variância genética entre as bacias leiteiras $i$ e $j$ ( $i$ e $j=1,2,3,4,5,6$ e 7, respectivamente). Nas matrizes $P$ e $R$, o índice $i$ identifica a bacia leiteira onde o animal foi observado.

Para a análise unicaracter, foi gerada uma cadeia de Gibbs de 120.000 iterações, com descarte inicial de 20.000 iterações e intervalo de amostragem de 100 iterações, obtendo-se 1.000 amostras de cada parâmetro estimado. Para as análises multicaracter, inicialmente foram geradas cadeias de 130.000 iterações, com descarte inicial de 30.000 iterações e intervalo de amostragem de 100 iterações. Cadeias 
de 100.000 foram acrescentadas até a amostragem atingir total de 830.000 iterações, obtendo-se 8.000 amostras de cada parâmetro estimado.

Os valores iniciais para as estimativas de co-variâncias genética aditiva, permanente de ambiente e residual para a raça Holandesa foram obtidos na literatura. Uma distribuição inicial uniforme foi assumida para os efeitos fixos considerando o parâmetro escala igual a 9 para os componentes de co-variância genética e o escala igual 3 para os componentes de variância permanente de ambiente.

A monitoração da convergência das cadeias geradas pelo amostrador de Gibbs foi feita utilizando-se os testes de diagnóstico de Geweke e de Heidelberger \& Welch, disponíveis no CODA (Convergence Diagnosis and Output Analysis), do programa R (2004) e verificando-se a convergência para todas as cadeias geradas.
Foram construídos os intervalos de credibilidade para todos os componentes de co-variância e parâmetros genéticos estimados no nível de $90 \%$.

A conexão genética entre as bacias leiteiras foi avaliada utilizando-se o conceito de similaridade genética, definido por Rekaya et al. (2003) (Tabela 2).

A existência ou não de interação genótipo $\times$ ambiente foi avaliada por meio das correlações genéticas entre as bacias leiteiras. Calcularam-se as correlações de Pearson entre os valores genéticos preditos e as correlações de Spearman entre a classificação (com base nos valores genéticos preditos) dos touros com filhos em comum em pelo menos duas bacias leiteiras.

Com base no valor genético predito, foram selecionados 10, dos 103 touros comuns a todas as bacias leiteiras, para verificar possíveis alterações na classificação dos touros e no valor genético dos animais nas bacias.

Tabela 2 - Similaridade genética (acima da diagonal) e número de touros em comum (abaixo da diagonal) entre as bacias leiteiras

\begin{tabular}{lccccccc}
\hline Bacia leiteira & Castro & Carambeí & Witmarsum & Arapoti & Sul & Norte & Oeste \\
\hline Castro & & 0,90 & 0,75 & 0,87 & 0,66 & 0,75 & 0,58 \\
Carambeí & 614 & & 0,76 & 0,86 & 0,64 & 0,78 & 0,61 \\
Witmarsum & 372 & 379 & 317 & 0,71 & 0,71 & 0,74 & 0,63 \\
Arapoti & 528 & 511 & 225 & 258 & 0,61 & 0,72 \\
Sul & 293 & 289 & 270 & 335 & 228 & 0,72 & 0,51 \\
Norte & 378 & 382 & 197 & 233 & 166 & 205 \\
Oeste & 269 & 273 & & & & 0,61 \\
\hline
\end{tabular}

\section{Resultados e Discussão}

As produções de leite, em kg, ajustadas para os 305 dias de lactação (PL305) nas bacias leiteiras de Castro, Carambeí, Witmarsum, Arapoti, Sul, Norte e Oeste foram de $8.414 \pm 1.825 ; 8.481 \pm 2.010 ; 7.636 \pm 1.594 ; 7.850 \pm 1.795$ $8.617 \pm 2.050 ; 7.401 \pm 1.809 ;$ e $7.336 \pm 2.456$, respectivamente. Essas médias são altas em comparação à média do estado do Paraná, porém foram obtidas apenas de animais sob controle leiteiro oficial da APCBRH, que não representam a população do estado.

As estimativas dos componentes de variância e herdabilidade foram obtidas com grande precisão (Tabela 3 ), pois apresentaram baixo erro-padrão e se mantiveram dentro do intervalo de credibilidade calculado.

A média posterior da herdabilidade para PL305 obtida na análise unicaracter está dentro do intervalo observado por Falcão et al. (2006), utilizando a inferência Bayesiana, por Ribas et al. (1993), utilizando o método dos quadrados mínimos, e por Almeida et al. (1997), utilizando o método da Máxima Verossimilhança Restrita. Esses autores encontraram valores de herdabilidade para PL305 no estado do Paraná igual a 0,28;0,23 $\pm 0,05$ e 0,28, respectivamente.

Assim como na análise unicaracter, as estimativas obtidas pela análise multicaracter foram de grande precisão. As menores estimativas dos componentes de variância genética e permanente de ambiente para PL305 foram observadas na bacia leiteira de Witmarsum (Tabela 4). O maior valor de variância genética aditiva e o menor valor de variância residual foram observados na bacia leiteira do Oeste do Paraná, o que implica maior valor de herdabilidade.

As médias posteriores dos componentes de co-variância genética e das variâncias permanente de ambiente e residual obtidas neste trabalho foram maiores que as estimadas por Falcão et al. (2006) para o rebanho leiteiro do estado do Paraná. Esta variação é esperada, em conseqüência das diferenças genéticas da população, de ambiente, do tipo de análise e do método de estimação de componentes de co-variância e, principalmente, de amostragem.

As médias posteriores das correlações genéticas (Tabela 5) entre todas as bacias leiteiras variaram de 0,09 (entre Witmarsum e Oeste) a 0,57 (entre Carambeí e Arapoti) 
Tabela 3 - Estimativas dos componentes de variâncias genética, permanente de ambiente e residual e da herdabilidade para a produção de leite, em kg, com seus respectivos intervalos de credibilidade, no nível de 90\%, obtidas na análise unicaracter

\begin{tabular}{|c|c|c|}
\hline Parâmetro & Média posterior e erro-padrão & Intervalo de credibilidade \\
\hline Variância genética & $502.539 \pm 565,23$ & $472.490-531.564$ \\
\hline Variância permanente de ambiente & $348.232 \pm 425,17$ & $326.056-370.553$ \\
\hline Variância residual & $1.055 .227 \pm 187,86$ & $1.045 .167-1.064 .423$ \\
\hline Herdabilidade & $0,26 \pm 0,0002$ & $0,25-0,28$ \\
\hline
\end{tabular}

Tabela 4 - Estimativas dos componentes de variâncias genética, permanente de ambiente e residual para a produção de leite, em kg, com seus respectivos intervalos de credibilidade, no nível de $90 \%$, obtidas nas sete bacias leiteiras do Paraná

\begin{tabular}{|c|c|c|}
\hline Bacia leiteira & Média posterior e erro-padrão & Intervalo de credibilidade \\
\hline & -----_-----_----_- & ------ \\
\hline Castro & $540.636 \pm 302,07$ & $496.395-585.365$ \\
\hline Carambeí & $476.609 \pm 348,51$ & $426.081-529.529$ \\
\hline Witmarsum & $396.962 \pm 353,50$ & $345.162-449.701$ \\
\hline Arapoti & $514.156 \pm 366,14$ & $460.340-569.001$ \\
\hline Sul & $593.678 \pm 635,48$ & $500.477-690.729$ \\
\hline Norte & $540.936 \pm 573,09$ & $457.243-627.596$ \\
\hline \multirow[t]{2}{*}{ Oeste } & $788.018 \pm 997,63$ & $646.549-937.363$ \\
\hline & $-\mathrm{V}$ & \\
\hline Castro & $358.115 \pm 224,04$ & $325.349-391.056$ \\
\hline Carambeí & $406.105 \pm 287,15$ & $364.386-448.632$ \\
\hline Witmarsum & $252.256 \pm 264,61$ & $213.994-292.592$ \\
\hline Arapoti & $371.878 \pm 275,14$ & $331.519-412.788$ \\
\hline Sul & $382.302 \pm 499,02$ & $309.533-457.145$ \\
\hline Norte & $377.309 \pm 452,07$ & $310.467-444.360$ \\
\hline \multirow[t]{2}{*}{ Oeste } & $497.817 \pm 769,38$ & $389.867-618.605$ \\
\hline & - & ------ \\
\hline Castro & $1.127 .537 \pm 110,29$ & $1.111 .452-1.144 .013$ \\
\hline Carambeí & $1.154 .214 \pm 147,19$ & $1.132 .629-1.175 .977$ \\
\hline Witmarsum & $782.710 \pm 148,12$ & $761.176-804.787$ \\
\hline Arapoti & $932.703 \pm 125,34$ & $914.059-951.163$ \\
\hline Sul & $1.066 .257 \pm 302,02$ & $1.022 .310-1.111 .387$ \\
\hline Norte & $946.253 \pm 266,01$ & $908.030-986.653$ \\
\hline Oeste & $719.761 \pm 375,92$ & $666.643-776.769$ \\
\hline
\end{tabular}

Tabela 5 - Médias posteriores da herdabilidade (na diagonal), da correlação genética (acima da diagonal) e dos componentes de co-variância genética (abaixo da diagonal) para a produção de leite, nas sete bacias leiteiras do Paraná

\begin{tabular}{|c|c|c|c|c|c|c|c|}
\hline Bacia leiteira & Castro & Carambeí & Witmarsum & Arapoti & Sul & Norte & Oeste \\
\hline Castro & 0,27 & 0,50 & 0,41 & 0,53 & 0,25 & 0,27 & 0,12 \\
\hline Carambeí & 254.600 & 0,23 & 0,38 & 0,57 & 0,22 & 0,29 & 0,11 \\
\hline Witmarsum & 188.494 & 164.010 & 0,28 & 0,35 & 0,20 & 0,21 & 0,09 \\
\hline Arapoti & 281.222 & 280.256 & 158.763 & 0,28 & 0,28 & 0,32 & 0,12 \\
\hline Sul & 140.154 & 119.232 & 95.999 & 152.703 & 0,29 & 0,14 & 0,10 \\
\hline Norte & 148.054 & 145.248 & 94.845 & 168.858 & 78.304 & 0,29 & 0,10 \\
\hline Oeste & 78.518 & 66.621 & 55.331 & 75.880 & 68.585 & 65.264 & 0,39 \\
\hline
\end{tabular}

e podem ser consideradas baixas de acordo com o critério de Robertson (1959), segundo o qual existe interação genótipo $\times$ ambiente, quando a correlação genética para a mesma característica em ambientes diferentes é menor que 0,80 .

Segundo Toral et al. (2004), as correlações de Pearson e Spearman assumem grande importância quando se consi- dera que, em programas de seleção, apenas os indivíduos com os melhores valores genéticos são escolhidos para reprodução e, como os valores de correlação são baixos, um touro escolhido com base nas informações dos filhos criados em determinada região pode não ter o mesmo desempenho quando utilizado como reprodutor em outras regiões. 
As correlações de Pearson e Spearman (Tabela 6) foram altas para PL305 entre as bacias leiteiras de Castro, Carambeí, Witmarsum, Arapoti, Sul e Norte, exceto para a bacia leiteira do Oeste, que, em comparação às outras, apresentou correlações de Pearson que variaram de 0,37 a 0,41 e de Spearman de 0,37 a 0,49. Esses resultados indicam interação genótipo $\times$ ambiente e confirmam a hipótese de que a classificação dos touros varia entre regiões. Isso induziria à escolha incorreta de touros e ocasionaria perda na eficiência de obtenção de ganho genético.

Houve coincidência na classificação de alguns touros entre a bacia leiteira de Castro e as de Carambeí, Witmarsum e Arapoti, que apresentaram alta correlação de Spearman, entretanto o valor genético dos touros não foi o mesmo em nenhuma região (Tabela 7). A bacia leiteira do Oeste apresentou a maior alteração na classificação e no valor genético dos touros (inclusive com valores genéticos negativos). Esses resultados podem ser explicados pelo fato de a bacia leiteira de Oeste ter sido estabelecida mais recentemente, com a introdução de animais de outras bacias leiteiras. Assim a bacia leiteira de Oeste se configura como um novo ambiente, diferente daquele onde o rebanho era criado. Uma evidência que pode suportar essa explicação é o fato de a herdabilidade na bacia leiteira do Oeste ser maior que as demais.

O touro número 1767 ficou classificado entre os dez melhores em todas as bacias leiteiras, entretanto sua classificação mudou em cinco das sete bacias estudadas e o

Tabela 6 - Correlações de Pearson entre os valores genéticos preditos (acima da diagonal) e de Spearman entre a classificação dos touros com base nos valores genéticos preditos (abaixo da diagonal) para a produção de leite nas sete bacias leiteiras do Paraná

\begin{tabular}{|c|c|c|c|c|c|c|c|}
\hline Bacia leiteira & Castro & Carambeí & Witmarsum & Arapoti & Sul & Norte & Oeste \\
\hline Castro & & $0,78 * *$ & $0,75 * *$ & $0,80 * *$ & $0,59 * *$ & $0,62 * *$ & $0,40 * *$ \\
\hline Carambeí & $0,77 * *$ & & $0,73 * *$ & $0,86^{* *}$ & $0,58 * *$ & $0,66 * *$ & $0,38 * *$ \\
\hline Arapoti & $0,77 * *$ & $0,84 * *$ & $0,69 * *$ & & $0,65 * *$ & $0,71 * *$ & $0,38 * *$ \\
\hline Sul & $0,59 * *$ & $0,60 * *$ & $0,59 * *$ & $0,66 * *$ & & $0,50 * *$ & $0,43 * *$ \\
\hline Norte & $0,61 * *$ & $0,64 * *$ & $0,56 * *$ & $0,68 * *$ & $0,49 * *$ & & $0,41 * *$ \\
\hline Oeste & $0,43 * *$ & $0,40 * *$ & $0,39 * *$ & $0,37 * *$ & $0,49 * *$ & $0,40 * *$ & \\
\hline
\end{tabular}

** Correlação significativa $(\mathrm{P}<0,01)$.

Tabela 7 - Valores genéticos preditos para a produção de leite de dez touros comuns a todas as bacias leiteiras e classificação dos touros (Classif.) em cada bacia leiteira

\begin{tabular}{|c|c|c|c|c|c|c|c|c|}
\hline \multirow{2}{*}{$\begin{array}{l}\text { Identificação do } \\
\text { reprodutor }\end{array}$} & \multicolumn{2}{|c|}{ Castro } & \multicolumn{2}{|c|}{ Carambeí } & \multicolumn{2}{|c|}{ Witmarsum } & \multicolumn{2}{|c|}{ Arapoti } \\
\hline & Classif. & Valor genético & Classif. & Valor genético & Classif. & Valor genético & Classif. & Valor genético \\
\hline 1926 & 1 & 1.951 & 7 & 1.037 & 1 & 1.204 & 9 & 1.089 \\
\hline 2264 & 2 & 1.733 & 14 & 800 & 10 & 781 & 10 & 1.009 \\
\hline 2361 & 3 & 1.666 & 1 & 1.575 & 12 & 666 & 3 & 1.508 \\
\hline 2238 & 4 & 1.370 & 2 & 1.411 & 19 & 510 & 1 & 1.816 \\
\hline 2798 & 5 & 1.297 & 18 & 672 & 14 & 575 & 16 & 688 \\
\hline 1767 & 6 & 1.214 & 6 & 1.065 & 5 & 865 & 2 & 1.525 \\
\hline 2585 & 7 & 1.195 & 3 & 1.287 & 24 & 424 & 8 & 1.219 \\
\hline 1713 & 8 & 1.191 & 4 & 1.234 & 2 & 1132 & 4 & 1.436 \\
\hline 2374 & 9 & 1.173 & 13 & 804 & 32 & 331 & 18 & 648 \\
\hline 2142 & 10 & 1.163 & 12 & 813 & 6 & 861 & 19 & 644 \\
\hline Identificação do & & & & & & & & \\
\hline reprodutor & Classif. & Valor genético & Classif. & Valor genético & Classif. & Valor genético & & \\
\hline 1926 & 42 & 230 & 52 & 61 & 4 & 892 & & \\
\hline 2264 & 4 & 870 & 43 & 125 & 60 & -28 & & \\
\hline 2361 & 1 & 1.050 & 8 & 871 & 61 & -38 & & \\
\hline 2238 & 11 & 751 & 3 & 1.178 & 12 & 557 & & \\
\hline 2798 & 27 & 356 & 9 & 851 & 21 & 344 & & \\
\hline 1767 & 8 & 802 & 2 & 1.220 & 2 & 1.025 & & \\
\hline 2585 & 28 & 347 & 14 & 696 & 18 & 413 & & \\
\hline 1713 & 20 & 548 & 11 & 768 & 55 & $-2,9$ & & \\
\hline 2374 & 48 & 174 & 30 & 278 & 94 & -457 & & \\
\hline 2142 & 7 & 828 & 5 & 1.143 & 41 & 108 & & \\
\hline
\end{tabular}


valor genético desse touro foi diferente em todas as bacias, o que caracteriza interação genótipo $\times$ ambiente e comprova que a interação genótipo $\times$ ambiente altera a classificação dos animais e pode levar à escolha inadequada de touros em determinadas regiões, prejudicando o melhoramento genético dos rebanhos.

Os resultados confirmam que, na avaliação genética, deve ser considerada a existência de interação genótipo $x$ ambiente para permitir a escolha adequada dos reprodutores a serem utilizados nas diferentes regiões.

\section{Conclusões}

Existe interação genótipo $\times$ ambiente para produção de leite em bovinos da raça Holandesa no estado do Paraná, principalmente quando se compara a bacia leiteira do Oeste às demais bacias, o que acarreta seleção equivocada dos melhores touros em cada região.

\section{Agradecimentos}

À Associação Paranaense de Criadores de Bovinos da Raça Holandesa, pela concessão do banco de dados.

À Coordenação de Aperfeiçoamento de Pesquisa de Nível Superior - CAPES, pela viabilização da pesquisa.

\section{Literatura Citada}

ALENCAR, M.M.; MASCIOLI, A.S.; FREITAS, A.R. Evidências de interação genótipo $\mathrm{x}$ ambiente sobre características de crescimento em bovinos de corte. Revista Brasileira de Zootecnia, v.34, n.2, p.489-495, 2005.

ALMEIDA, R.; RIBAS, N.P.; MONARDES, H.G. Estudo dos efeitos genéticos sobre as características produtivas de vacas da raça Holandesa na região da Batavo, Paraná. In: REUNIÃO ANUAL DA SOCIEDADE BRASILEIRA DE ZOOTECNIA, 34., 1997, Juiz de Fora. Anais... Juiz de Fora: Sociedade Brasileira de Zootecnia, v.3, 1997. p.68-70.

CERÓN-MUÑOZ, M.F.; TONHATI, H.; COSTA, C.N. Factors that cause genotype by environment interaction and use of a multiple-trait herd-cluster model for milk yield of Holstein cattle from Brazil and Colombia. Journal of Dairy Science, v.87, p.2687-2692, 2004.
COSTA, C.N.; BLAKE, R.W.; POLLAK, E.J. et al. Genetic analysis of Holstein cattle populations in Brazil and the United States. Journal of Dairy Science, v.83, p.2963-2974, 2000.

FALCÃO, A.J.S.; MARTINS, E.N.; COSTA, C.N. et al. Heterocedasticidade entre estados para produção de leite em vacas da raça Holandesa usando métodos bayesianos via amostrador de Gibbs. Revista Brasileira de Zootecnia, v.35, n.2, p.405-414, 2006.

R Development Core Team [2004]. R: A language and environment for statistical computing. Vienna: R Foundation for Statistical Computing. Disponível em: <http://www.R-project.org.> Acesso em: $10 / 12 / 2005$.

REKAYA, R.; WEIGEL, K.A.; GIANOLA, D. Bayesian estimation of parameters of a structural model for genetic covariances between milk yield in five regions of the United States. Journal of Dairy Science, v.86, p.1837-1844, 2003.

RIBAS, N.P.; RORATO, P.R.N.; LÔBO, R.B. et al. Estimativas de parâmetros genéticos para as características de produção da raça holandesa no Estado do Paraná. Revista Brasileira de Zootecnia, v.22, n.4, p.634-641, 1993.

ROBERTSON, A. The sampling variance of the genetic correlation coefficient. Biometrics, v.15, p.469, 1959.

STATISTICAL ANALYSIS SYSTEM - SAS. SAS/STAT ${ }^{o ̀: ~ u s e r ' s ~}$ guide: statistics. versão 8.1. 4.ed. Cary: SAS Institute, 2000. v.2. (CD-ROM).

SEAB-DERAL. Caracterização da bovinocultura de leite no estado do Paraná. Curitiba: Governo do estado do Paraná, Secretaria do Estado da Agricultura e do Abastecimento (SEAB), Departamento de Economia Rural (DERAL), Divisão de Conjuntura Agropecuária (DCA), 2000. Disponível em: <http:// www.pr.gov.br/seab/deral/cultura3.pdf.> Acesso em: 5/1/2006.

SEAB-DERAL. Análise da conjuntura agropecuária safra 2007/2008 - Leite. Curitiba: Governo do estado do Paraná, Secretaria do Estado da Agricultura e do Abastecimento (SEAB), Departamento de Economia Rural (DERAL), 2007. Disponível em:<http://www.seab.pr.gov.br/arquivos/File/deral/Prognosticos/ leite_2007_08.pdf.> Acesso em: 1/12/2008.

STANTON, T.L.; BLAKE, R.W.; QUAAS, R.L. genotype by environment interaction for Holstein milk yield in Colombia, Mexico, and Puerto Rico. Journal of Dairy Science, v.74, p.1700-1714, 1991.

TORAL, F.L.B.; SILVA, L.O.C.; MARTINS, E.N. et al. Interação genótipo $\mathrm{x}$ ambiente em características de crescimento de bovinos da raça Nelore no Mato Grosso do Sul. Revista Brasileira de Zootecnia, v.33, n.6, p.1445-1455, 2004.

Van TASSEL, C.P.; Van VLECK, D.L. A manual for use of MTGSAM. A set of FORTRAN programs to apply Gibbs sampling to animal models for variance component estimation (DRAFT). Lincoln: Department of Agriculture Research Service, 1995. 86p.

ZWALD, N.R.; WEIGEL, K.A.; FIKSE, W.F. et al. Identification of factors that cause genotype by environment interaction between herds of Holstein cattle in seventeen countries. Journal of Dairy Science, v.86, p.1009-1018, 2003. 